\title{
A novel role for ribonuclease 9 in suppressing sperm capacitation and acrosome reaction
}

\author{
Jie $\mathrm{Liu}^{1}$, Yuanyuan $\mathrm{Hou}^{2}$, Lei Gong ${ }^{1}$, Yuanyuan Gu${ }^{2}$, Lijun Duan ${ }^{1}$, Yuxiao Tang ${ }^{3}$, Wenting Wang ${ }^{1 *}$ \\ ${ }^{1}$ Biochip Laboratory, Yantai Yuhuangding Hospital affiliated to Qingdao University, Yantai 264000 China \\ 2 Obstetrics Department, Yantai Yuhuangding Hospital affiliated to Qingdao University, Yantai 264000 \\ China \\ 3 Endocrine Department, Yantai Yuhuangding Hospital affiliated to Qingdao Universit, Yantai 264000 \\ China
}

*Corresponding author, e-mail: sss_wen678@163.com

Received 28 Apr 2018

Accepted 17 Dec 2018

\begin{abstract}
Ribonuclease 9 (RNase 9) is a member of the ribonuclease A superfamily, which is mainly expressed in the epididymis. However, it has no ribonucleolytic activity and its function remains unclear. Immunofluorescence experiments showed that the localization of RNase 9 protein on the sperm surface has moved from the neck to the acrosome cap during capacitation and acrosome reaction. Chlortetracycline staining was used to assay the role of RNase 9 on capacitation and acrosome reaction. The spontaneous acrosome reaction induced by progesterone could be suppressed by RNase 9 protein and the acrosome reaction ratio was $6.5 \pm 1.2 \%$. Furthermore, exposure of spermatozoa to RNase 9 protein reduced the level of cyclic AMP significantly during the acrosome reaction. Taken together, we confirmed that RNase 9 protein could impair sperm function, including capacitation and acrosome reaction, and had potential role in male reproductive toxicity.
\end{abstract}

KEYWORDS: immunofluorescence, chlortetracycline staining, cyclic AMP

\section{INTRODUCTION}

The ribonuclease A (RNase A) superfamily is a vertebrate-specific family with 13 members. One of these members, RNase 9, is mainly expressed in the endothelial cells of epididymis and is localized on the posterior equatorial region of the sperm ${ }^{1-3}$. The work of Cheng et al showed that recombinant human RNase 9 did not exhibit detectable ribonucleolytic activity but exhibited antibacterial activity ${ }^{4}$. However, the influence of RNase 9 on the maturation, motility and fertilization of sperm is still unclear.

Acrosome reaction refers to a series of changes in the acrosome during fertilization, including the process of releasing acrosin from spermatozoa and eroding the corona radiata and zona pellucida (ZP). This process is very important in fertilization that allows the sperm passage through ZP and then to fuse with egg yolk ${ }^{5}$.

In mammals, the sperm generally stay in the oviduct for a relatively long period of time to gain full fertility. During this period, the sperm undergoes a complex process called capacitation ${ }^{6}$. This process requires the activation of cyclic AMP (cAMP) and $\mathrm{Ca}^{2+}$ signalling, as well as the increase in the level of tryptophan phosphorylation ${ }^{7-9}$. In particular, the regulation of cAMP level is crucial during fertilization since cAMP could activate different signalling pathways, such as $\mathrm{Ca}^{2+}$ level, phospholipase C (PLC), PKC/ERK1-2, as well as soluble AC and cAMP/protein kinase A (PKA) signalling ${ }^{10}$. Capacitation usually includes biochemical and morphological changes. Biochemical changes involve the removal or redistribution of epididymal proteins and seminal plasma proteins on the surface of spermatozoa, changes in membrane lipid composition, membrane protein migration, and receptor exposure ${ }^{11}$. As RNase 9 is one of the sperm surface proteins, the involvement of this protein in sperm capacitation, acrosome reaction and fertilization is still unclear. Thus in this study, we demonstrated that RNase 9 could impair capacitation and acrosome reaction of sperms. Furthermore, we provide supporting evidence for its potential role in male reproductive toxicity. 


\section{MATERIALS AND METHODS}

\section{Sperm collection}

Semen samples were collected from 20 to 40 -yearold healthy donors. All donors were recruited under informed consent after approval by the Institutional Review Board of Yantai Yuhuangding Hospital affiliated to Qingdao University. Sperms were obtained by masturbation after 3-6 days of sexual abstinence. All samples were collected in $5 \mathrm{ml}$ disposable containers and left for at least $30 \mathrm{~min}$ to liquefy. The concentration, volume, morphology and viability of sperms were analysed according to the previous study ${ }^{12}$. Parameters of the sperms, including semen volume, $\mathrm{pH}$, morphology, progressive motility (PR) and concentration, were classified to be normal, according to the criteria of World Health Organization (WHO, 2010). The sperms were separated by 45-90\% percoll gradient centrifugation (Pharmacia, USA ${ }^{13}$. Sperms were washed 3 times at $700 \mathrm{~g}$ for $18 \mathrm{~min}$ and resuspended with Biggers, Whitten, and Whittingham (BWW) medium (Sigma). Sperms were adjusted to a concentration of $10^{6}$ spermatozoa per $\mathrm{ml}$, then incubated at $37^{\circ} \mathrm{C}$ with $5 \% \mathrm{CO}_{2}$ before further use.

\section{Immunofluorescence staining}

Sperms were incubated in BWW medium for 0 , 3 , and $5 \mathrm{~h}$ at $37^{\circ} \mathrm{C}$ with $5 \% \mathrm{CO}_{2}$. Progesterone (Sigma, USA) was freshly prepared as a stock solution (2 mM) in dimethyl sulphoxide (DMSO). After capacitation, progesterone was added to the sperms at a final concentration of $20 \mu \mathrm{M}$, and incubated for $30 \mathrm{~min}$ at $37^{\circ} \mathrm{C}$. The sperm suspension were spotted onto slides, dried at room temperature, and fixed in 4\% paraformaldehyde for $15 \mathrm{~min}$. The samples were added to PBS buffer with $3 \%$ bovine serum albumin (BSA) and incubated with a 1:400 dilution of mouse polyclonal antibody overnight at $4{ }^{\circ} \mathrm{C}$. Then they were incubated with goat antimouse IgG-labelled FITC (1:2000 dilution) at room temperature for $1 \mathrm{~h}$. The negative control was incubated in PBS buffer with 3\% BSA. Sperm nuclei were stained with propidium iodide (Sigma), observed under confocal laser scanning microscope (LSM710; Zeiss, Germany) and analysed using the software Image-Pro Plus 5.1 (Media Cybernetics, USA).

\section{Chlortetracycline (CTC) staining}

The swimming-up sperm suspensions were divided into three aliquots containing at least $10 \times 10^{6}$ spermatozoa per $\mathrm{ml}$. These sperms were co-cultured with RNase $9(0.8 \mu \mathrm{g} / \mathrm{ml}$; Origene, USA), antiRNase 9 (1:500; Origene, USA) or PBS containing $3 \%$ BSA in BWW medium for $5 \mathrm{~h}$, respectively. CTC staining was performed in this study and three types of CTC fluorescent staining (F, B and AR) were found. F pattern was for uncapacitated spermatozoa with intact acrosomal cap as shown by a strong yellow-green fluorescence signal, B pattern was for capacitated spermatozoa with complete acrosomal cap and only fluorescent signal in the posterior region of the acrosome cap, whereas AR pattern displayed light green colour or no fluorescence at the acrosome region. At least 200 spermatozoa were counted in each smear. Three independent experiments were performed in duplicate and the percentage of F, B and AR patterns were calculated.

\section{cAMP measurement}

The swimming-up sperm suspensions were divided into three aliquots containing at least $10 \times 10^{6}$ spermatozoa per $\mathrm{ml}$. These sperms were co-cultured with RNase $9(0.8 \mu \mathrm{g} / \mathrm{ml})$, anti-RNase 9 (1:500, Origene) or PBS containing 3\% BSA in BWW medium for $5 \mathrm{~h}$, respectively. The spermatozoa were separated and the pellets were homogenized in $0.1 \mathrm{M}$ $\mathrm{HCl}$. The homogenates were centrifuged at $10000 \mathrm{~g}$ at room temperature for $30 \mathrm{~min}$. The supernatants were acetylated before determining the cAMP concentrations following manufacturer's instructions (Cyclic AMP Complete ELISA Kit, Abcam, USA). cAMP levels were calculated in femtomole of cAMP per $10^{6}$ spermatozoa.

\section{Statistical analysis}

SPSS (SPSS standard version 13.0) was used for statistical analyses. Values are presented as mean \pm SD. Student's $t$-test was used to compare different groups. A $p$-value less than 0.05 was considered to be statistically significant.

\section{RESULTS}

Localization of RNase 9 during capacitation and acrosome reaction

RNase 9 protein was localized in the head-neck region of normal sperms. During the capacitation process, RNase 9 protein migrated from the headneck to the equatorial region on the sperms. The green fluorescence staining gradually weakened on the head-neck region of sperms at $3 \mathrm{~h}$ of capacitation (Fig. 1 (a)3, (b)3, and (c)3). At 5 h of capacitation, there were obvious visible green fluorescence bands at the equatorial region and the RNase 9 protein 


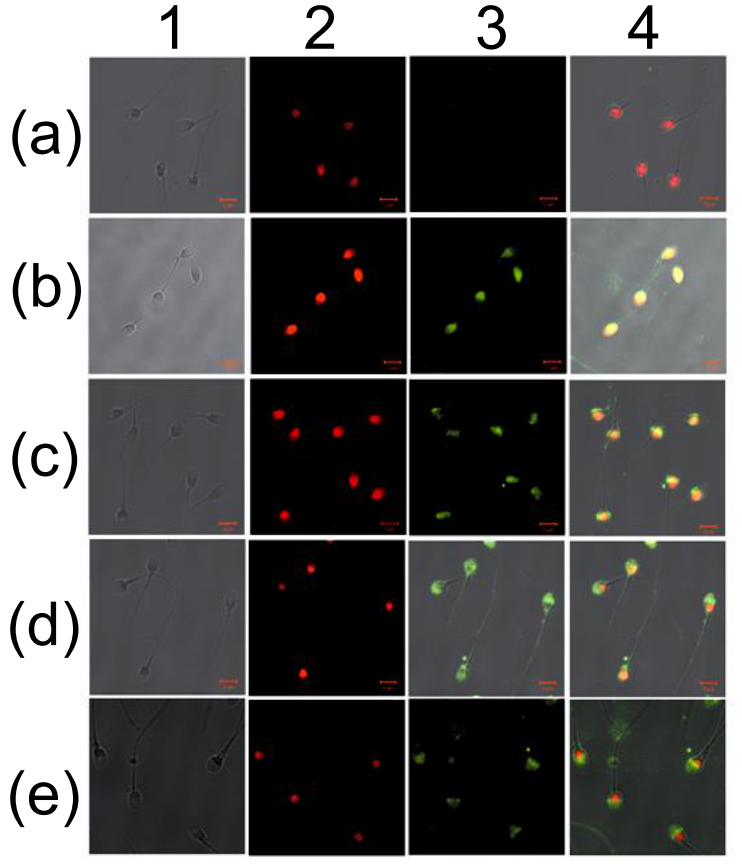

Fig. 1 Immunofluorescence staining of RNase 9 protein. (a) negative control (PBS with 3\% BSA); (b) precapacitation; (c) capacitation for $3 \mathrm{~h}$; (d) capacitation for $5 \mathrm{~h}$; (e) acrosome reaction. (a)1-(e)1 black and white images of sperms; (a)2-(e)2 nuclear staining of sperms (red fluorescent); (a)3-(e)3 immunofluorescent staining of RNase 9 protein (green fluorescent); (a)4-(e)4 merged images. Green and red fluorescent signals represent the location of RNase 9 protein and the nucleus, respectively.

further migrated from the equatorial region to the acrosome and covered the whole acrosomal cap (Fig. 1 (a)3, (b)3, and (d)3). During the course of progesterone induced-acrosome reaction, the colour of the sperm acrosome was found to be less intense, suggesting that acrosin might be released. Weak green fluorescence signal was observed in the equatorial region (Fig. 1 (a)3, (b)3, and (e)3).

\section{Effects of RNase 9 on capacitation and acrosome reaction}

Immunofluorescence experiments showed that in the process of capacitation and acrosome reaction, the localization of the RNase 9 protein on the sperm surface was redistributed. Whether RNase 9 protein is involved in capacitation and acrosome reaction needs to be further studied. A significantly lower incidence rate of spontaneous acrosome reaction $(6.5 \pm 1.2 \% ; p<0.05)$ was observed when sperms were co-cultured with RNase 9 protein $(0.8 \mu \mathrm{g} / \mathrm{ml})$

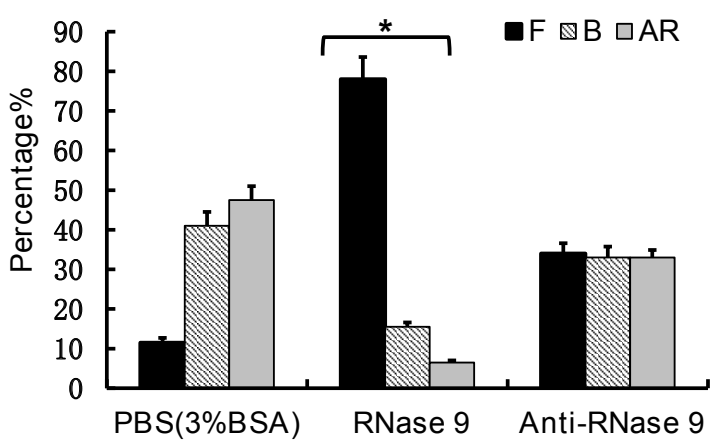

Fig. 2 Effects on capacitation and acrosome reaction. Three independent experiments were performed in duplicate and the values are mean $\pm \mathrm{SD}, * p<0.05$.

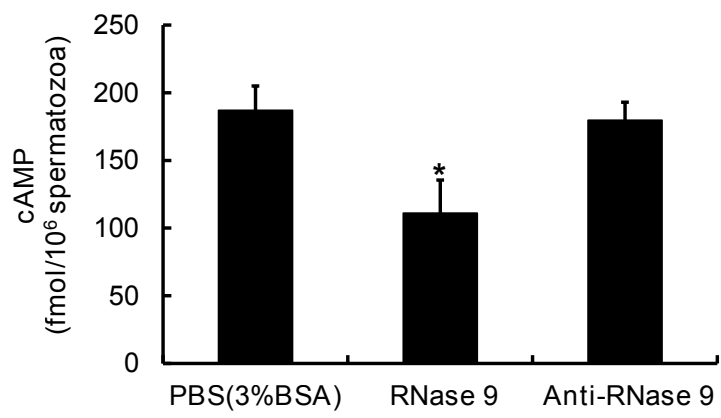

Fig. 3 cAMP levels during the acrosome reaction. Three independent experiments were performed in duplicate and the values are mean $\pm \mathrm{SD},{ }^{*} p<0.05$.

in BWW medium and the F pattern rate is $78 \pm 5.6 \%$ (Fig. 2). However, anti-RNase 9 had no effect on sperm capacitation and acrosome reaction, compared to the PBS (3\% BSA) group.

\section{Levels of intracellular cAMP}

Capacitation of sperms in the female reproductive tract is the key prerequisite to acquire the ability of the sperms to fertilize eggs. cAMP also plays pivotal roles in molecular events leading to mammalian capacitation and acrosome reaction. This prompted us to measure cAMP levels in capacitated or acrosomereacted spermatozoa. The exposure of spermatozoa to RNase 9 protein $(0.8 \mu \mathrm{g} / \mathrm{ml})$ produced a significant decrease in the intracellular cAMP levels during acrosome reaction when compared to those of control $\left(111 \pm 24\right.$ versus $187 \pm 18 \mathrm{fmol} / 10^{6}$ spermatozoa; $p<0.05$ ) (Fig. 3). However, the treatment of spermatozoa with anti-RNase 9 produced no significant changes in intracellular cAMP levels when 
compared to those of control samples $(180 \pm 13$ and $187 \pm 18$ fmol $/ 10^{6}$ spermatozoa; $p<0.05$ ) (Fig. 3).

\section{DISCUSSION}

At present, thirteen members of the RNase A superfamily have been found in humans. RNase 9-13 are newly discovered members in male reproductive organs ${ }^{14-16}$. The specific expressions of RNase 9 and RNase 10 in the epididymis have been reported. However, the functions of these proteins are still unclear ${ }^{14}$. Mammalian sperms in the female reproductive tract can have the capability to fertilize after undergoing biochemical and morphological changes. Sperm capacitation is a multistep process and is an important physiological prerequisite for acrosome reaction, hyper activation movement and fertilization ${ }^{17}$. Capacitation involves changes in sperm membrane proteins and changes in membrane fluidity until the acrosome reaction occurs. The molecular mechanism of sperm capacitation is quite complex and is not yet fully defined. In this study, we found that the subcellular localization of RNase 9 on the sperm surface changed or redistributed during capacitation. This result suggested that RNase 9 protein might be involved in the capacitation process. Furthermore, the result from CTC staining demonstrated that RNase 9 protein inhibited sperm capacitation and acrosome reaction. Consistent with the result of CTC staining, the levels of cAMP in sperm capacitation was inhibited in the presence of RNase 9 protein. These experiments confirmed that RNase 9 protein significantly impaired sperm function, including capacitation and acrosome reaction, and provide evidence for its potential role in male reproductive toxicity.

Acknowledgements: We thank all the participants who took part in this study. This work was supported by the National Natural Science Foundation of China (Grant No. 81501314).

\section{REFERENCES}

1. Castella S, Fouchecourt S, Teixeira-Gomes AP, Vinh J, Belghazi M, Dacheux F, Dacheux JL (2004) Identification of a member of a new RNase a family specifically secreted by epididymal caput epithelium. Biol Reprod 70, 319-28.

2. Penttinen J, Pujianto DA, Sipila P, Huhtaniemi I, Poutanen M (2003) Discovery in silico and characterization in vitro of novel genes exclusively expressed in the mouse epididymis. Mol Endocrinol 17, 2138-51.

3. Cho S, Beintema JJ, Zhang J (2005) The ribonuclease A superfamily of mammals and birds: identifying new members and tracing evolutionary histories. Genomics 85, 208-20.

4. Cheng GZ, Li JY, Li F, Wang HY, Shi GX (2009) Human ribonuclease 9, a member of ribonuclease A superfamily, specifically expressed in epididymis, is a novel sperm-binding protein. Asian J Androl 11, 240-51.

5. Ickowicz D, Finkelstein M, Breitbart H (2012) Mechanism of sperm capacitation and the acrosome reaction: role of protein kinases. Asian J Androl 14, 816-21.

6. O'Flaherty C (2015) Redox regulation of mammalian sperm capacitation. Asian J Androl 17, 583-90.

7. Visconti PE, Moore GD, Bailey JL, Leclerc P, Connors SA, Pan D, Olds-Clarke P, Kopf GS (1995) Capacitation of mouse spermatozoa. II. Protein tyrosine phosphorylation and capacitation are regulated by a cAMP-dependent pathway. Development 121, 1139-50.

8. Salicioni AM, Platt MD, Wertheimer EV, Arcelay E, Allaire A, Sosnik J, Visconti PE (2007) Signalling pathways involved in sperm capacitation. Soc Reprod Fertil Suppl 65, 245-59.

9. Li X, Wang L, Li Y, Zhao N, Zhen L, Fu J, Yang Q (2016) Calcium regulates motility and protein phosphorylation by changing cAMP and ATP concentrations in boar sperm in vitro. Anim Reprod Sci 172, 39-51.

10. Alonso CAI, Osycka-Salut CE, Castellano L, Cesari A, Di Siervi N, Mutto A, Johannisson A, Morrell JM, et al (2017) Extracellular cAMP activates molecular signalling pathways associated with sperm capacitation in bovines. Mol Hum Reprod 23, 521-34.

11. Botto L, Bernabo N, Palestini P, Barboni B (2010) Bicarbonate induces membrane reorganization and CBR1 and TRPV1 endocannabinoid receptor migration in lipid microdomains in capacitating boar spermatozoa. J Membr Biol 238, 33-41.

12. Lu JC, Huang YF, Lu NQ (2014) Computer-aided sperm analysis: past, present and future. Andrologia 46, 329-38.

13. Zhou JH, Zhou QZ, Lyu XM, Zhu T, Chen ZJ, Chen MK, Xia H, Wang CY, et al (2015) The expression of cysteine-rich secretory protein 2 (CRISP2) and its specific regulator miR-27b in the spermatozoa of patients with asthenozoospermia. Biol Reprod 92, $1-9$.

14. Krutskikh A, Poliandri A, Cabrera-Sharp V, Dacheux JL, Poutanen M, Huhtaniemi I (2012) Epididymal protein Rnase10 is required for post-testicular sperm maturation and male fertility. FASEB J 26, 4198-209.

15. Yamada Y, Sakuma J, Takeuchi I, Yasukochi Y, Kato K, Oguri M, Fujimaki T, Horibe H, et al (2017) Identification of EGFLAM, SPATC1L and RNASE13 as novel susceptibility loci for aortic aneurysm in Japanese individuals by exome-wide association studies. Int $J$ Mol Med 39, 1091-100. 
16. Mukherjee S, Kim S, Ramanan VK, Gibbons LE, Nho K, Glymour MM, Ertekin-Taner N, Montine TJ, et al (2014) Gene-based GWAS and biological pathway analysis of the resilience of executive functioning. Brain Imaging Behav 8, 110-8.

17. Stival C, Puga Molina LC, Paudel B, Buffone MG, Visconti PE, Krapf D (2016) Sperm capacitation and acrosome reaction in mammalian sperm. In: Buffone MG (ed) Sperm Acrosome Biogenesis and Function During Fertilization, Advances in Anatomy, Embryology and Cell Biology 220, Springer, Cham, pp 93-106. 